\title{
CMOS Spectrally-Multiplexed FRET-on-a-Chip for DNA Analysis
}

\author{
Derek Ho, Student Member, IEEE, M. Omair Noor, Ulrich J. Krull, Glenn Gulak, Senior Member, IEEE, and \\ Roman Genov, Senior Member, IEEE
}

\begin{abstract}
A spectral-multiplexed fluorescence contact imaging microsystem for DNA analysis is presented. The microsystem integrates a filterless CMOS Color PhotoGate (CPG) sensor that exploits the polysilicon gate as an optical filter, and therefore does not require an external color filter. The CPG is applied to fluorescence-based transduction in a spectrally multiplexed format by differentiating among multiple emission bands, hence replacing the functionality of a bank of emission filters. A microsystem has been quantitatively modeled and prototyped based on the CPG fabricated in a standard $0.35 \mu \mathrm{m}$ CMOS technology. The multi-color imaging capability of the microsystem in analyzing DNA targets has been validated in the detection of marker gene sequences for spinal muscular atropy disease and Escherichia coli (E. coli). Spectral-multiplexing enables the two DNA targets to be simultaneously detected with a measured detection limits of $240 \mathrm{nM}$ and $210 \mathrm{nM}$ for the two target concentrations at a sample volume of $10 \mu \mathrm{L}$ for the green and red transduction channels, respectively.
\end{abstract}

Index Terms - Black hole quencher, CMOS image sensor, contact imaging, DNA detection, fluorescence, point-of-care diagnostics, quantum dot, spectral-multiplexing.

\section{INTRODUCTION}

$\mathbf{T}$ HE analysis of nucleic acids, such as deoxyribonucleic acid (DNA), is invaluable to many areas of the life sciences, for example, in cancer research, clinical diagnostics for genetic diseases and for pathogen detection in food [1]. DNAbased sensing technologies interrogate selective hybridization of complementary DNA sequences and are conventionally designed for parallel detection of multiple nucleic acid targets [2].

Although the conventional microarray technology employs spatial registration to achieve unprecedented parallelism, it suffers from disadvantages such as additional processing required to print spots on a surface and the associated spatial variation in terms of the quality of the probe immobilization across discrete spots [2], [3]. Also, the hybridization chemistry requires the washing away of samples after introducing them to the probes. This renders real-time sensing difficult, often prohibitive.

Manuscript received July 03, 2012; revised November 09, 2012; accepted November 17, 2012. Date of publication February 26, 2013; date of current version October 24, 2013. This paper was recommended by Associate Editor K. C. Cheung.

D. Ho, G. Gulak, and R. Genov are with the Department of Electrical and Computer Engineering, University of Toronto, Toronto, ON M5S 3G4, Canada (e-mail: hoderek@eecg.utoronto.ca; gulak@eecg.utoronto.ca; roman@eecg.utoronto.ca).

M. O. Noor and U. J. Krull are with the Department of Chemical and Physical Sciences, University of Toronto Mississauga, Mississauga, ON L5L 1C6, Canada (e-mail: omair.noor@utoronto.ca; ulrich.krull@utoronto.ca).

Color versions of one or more of the figures in this paper are available online at http://ieeexplore.ieee.org.

Digital Object Identifier 10.1109/TBCAS.2012.2230172
Therefore only the outcome rather than the dynamics of the biological experiment can be observed. In addition, microarray manufacturing typically involves time-consuming processes and a laboratory environment. They often offer much greater capabilities, such as parallelism, than required for many in-field applications such as pathogen detection [4].

An alternative to spatial registration is spectral multiplexing. In the case of fluorescence-based DNA detection, different DNA target sequences can be tagged with fluorophores that emit light at different wavelengths, which can be concurrently detected and distinguished. Unlike other spectroscopic techniques, such as Raman spectroscopy, where continuous fine spectral resolution is required, fluorescent imaging requires spectral differentiation among only a few discrete wavelengths [5], [6].

Conventionally, differentiation between fluorescent emission wavelengths has been achieved by using a set of optical bandpass filters to select different parts of the emission spectrum. The optics involved is bulky and expensive. To circumvent this problem, other spectral methods have also been investigated. Methods based on diffraction grating (the splitting of light) [7] and Fabry-Perot etalon (tuned resonance cavity) [8] generally offer high spectral resolution, but require micromachining and post-processing such as wafer polishing and wafer bonding. Eliminating the need for sophisticated optics and post-processing is the ultimate remedy to the high design complexity and fabrication cost.

Techniques that solely rely on integrated circuit process technology have been developed, most notably the buried junction technology [9], [10] (on which the Foveon sensor [11] is based). Since light absorption in a semiconductor varies across wavelengths in such a way that light of a longer wavelength can penetrate deeper, a photocurrent measured at a deeper depth consists of stronger long-wavelength components. By sensing at several depths, color information can be inferred. Although the buried junction approach achieves high spatial density and is suitable for photographic applications requiring three colors (e.g., blue, green, and red), there is a limit to the number of diodes that can be implemented, for example three for a dual-well process. This limits its support for applications that require sensing at more than three wavelengths. To overcome this limitation, a spectrally-sensitive photodiode that can potentially sense more than three colors has been prototyped [12]. A biased poly-silicon gate modulates the photo sensing region depth to effectively achieve an equivalent of many buried diodes. However, the reliance on the vertical dimensions of the CMOS process technology limits scalability. The most recently reported prototype is fabricated in a $5 \mu \mathrm{m}$ custom integrated circuit process [12]. 


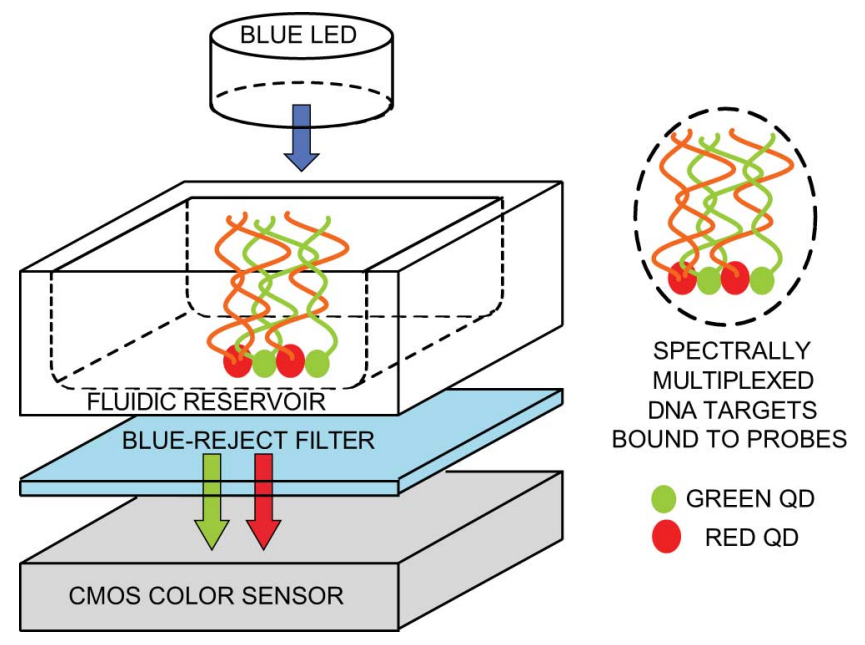

Fig. 1. CMOS fluorescent DNA contact imaging microsystem.

In recent years, point-of-care (POC) diagnostic devices have become increasingly popular as they promise to bring diagnostic technology from the standard laboratory setting to the patient residence to facilitate early diagnosis [13]. Optical transduction methods such as fluorescence-based detection have widespread applications such as detection of nucleic acids, proteins and small molecules. But despite the high sensitivity and selectivity offered by this method, its incorporation into POC devices has been limited due to the limited portability and high cost of the instrumentation, as a fluorescence microscope is often required. One emerging technique with a potential to overcome the limitations of a fluorescence microscope is contact imaging [14], which enables miniaturized biosensors [10], [15], [16]. Unlike the conventional fluorescent microscope, in contact imaging as depicted in Fig. 1, the object to be imaged is placed in close proximity to the focal plane. In this technique, the resolution of the imaging system is in the order of the pixel size. This technique eliminates the need for bulky and expensive optics such as a system of lenses and mirrors, which enables miniaturization to realize lab-on-a-chip platforms.

In this paper, we present a low-cost CMOS spectrally-multiplexed contact imaging microsystem for DNA analysis. The core of the microsystem is a CMOS color photogate (CPG) sensor, with an earlier generation prototype reported in [17]-[19]. The pixel consists of the CPG to differentiate among fluorescent biomarkers emission bands and a current-to-frequency analog-to-digital converter (ADC) for digital readout. Only one optical filter to attenuate the excitation light is required. The sensor is prototyped in a standard digital $0.35 \mu \mathrm{m}$ CMOS technology and experimentally validated in the simultaneous detection of two DNA targets, the SMN1 and uidA sequences, which diagnose the spinal muscular atropy disease and the $E$. coli bacteria, respectively.

The remainder of the paper is organized as follows. Section II discusses the DNA detection chemistry used in this work. Section III and IV present a mathematical model and a prototype of a fluorescent contact imaging system, respectively. Section $\mathrm{V}$ details the design and implementation of the CPG sensor. Section VI reports experimental validation of the CPG-based

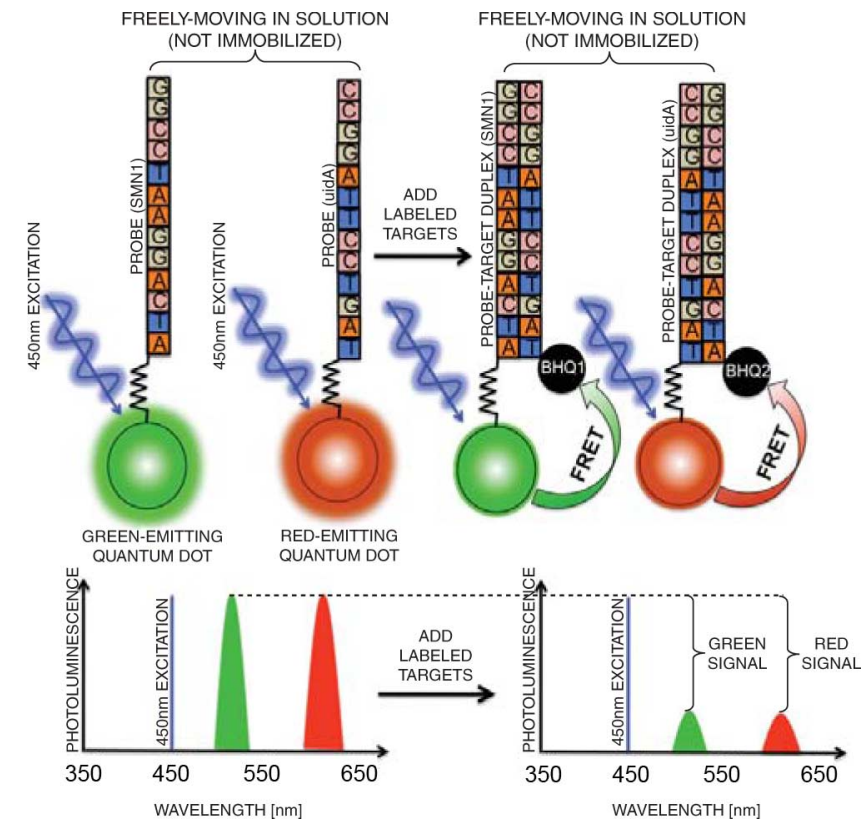

Fig. 2. Förster resonance energy transfer (FRET) based assay design for spectrally-multiplexed detection of DNA hybridization using multi-color QDs and black hole quencher (BHQ) labeled DNA targets. (top) Green-emitting QDs (gQDs) and red-emitting QDs (rQDs) are conjugated with SMN1 and uidA probes respectively. (bottom) Expected changes in the emission of gQDs and rQDs after hybridization. Note: a single excitation source can be used to excite both colors of QDs.

biosensor in fluorescence-based DNA detection. Section VII highlights key observations.

\section{DNA DETECTION CHEMISTRY}

In order to appreciate the requirements and functionality of the microsystem, the chemistry and the schematics of the assay design for a multiplexed detection of nucleic acid hybridization are depicted in Fig. 2. To interrogate the probe-target hybridization event, the assay employs multi-color colloidal semiconductor nanocrystals or quantum dots (QDs) as energy donors and targets labeled with black hole quenchers (BHQs) as acceptors in the Förster resonance energy transfer (FRET) process. FRET is a mechanism that transfers energy between two molecules. A donor molecule may transfer energy to an acceptor molecule, over a distance typically less than $10 \mathrm{~nm}$, through non-radiative dipole-dipole coupling [20].

QDs exhibit a number of unique optical properties that are advantageous for spectral multiplexing. These unique properties include: narrow, symmetric and size-tunable emission photoluminescence spectra (full width at half maximum, FWHM, 25-35 nm); strong and broad absorption spectra; high quantum yields $(>20 \%)$ and long life times (>10 ns) [21]. As compared to organic fluorophores, QDs have greater resistance to photobleaching that enables long-term monitoring. The broad absorption spectra of QDs allows for multiple colors of QDs to be excited efficiently with a single excitation source which is not possible with organic dyes. These optical properties of QDs make them suitable for optical multiplexing and as donors for FRET-based applications [20].

In the proposed assay, green-emitting QDs (gQDs) and red-emitting QDs (rQDs) are conjugated with the SMN1 
and uidA probes, respectively. The survival motor neuron 1 (SMN1) sequence is a diagnostic of neurodegenerative disease called spinal muscular atrophy. The uidA sequence is a marker for an E. coli bacteria. SMN1 targets are labeled with BHQ1 and uidA targets are labeled with BHQ2. Hence, the FRET pairs relevant to this work are gQDs/BHQ1 (donor/acceptor) and rQDs/BHQ2 (donor/acceptor). Upon introduction of labeled targets, the hybridization event brings the acceptors (BHQ1 and BHQ2) in close proximity to the $\mathrm{QD}$, which allows the BHQs to absorb a portion of the QD emission via the FRET process. This absorption of energy reduces or quenches the QD emission. This serves as the analytical signal to be detected. A higher target concentration implies more BHQs are present, resulting in a reduction in the overall observable QD emission signal.

The DNA probe and target sequences used are from Integrated DNA Technologies [22] with the following nucleobase composition for SMN1 and uidA, respectively

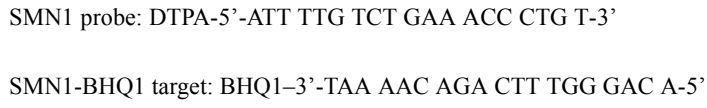

where DTPA denotes dithiol phosphoramidite, a chemical moiety used to immobilize the DNA probe sequences on the surface of QDs. Since a donor QD and an acceptor BHQ form a FRET pair for detecting a particular target DNA analyte, it is possible to employ different colors of the QD/BHQ FRET pair to detect multiple target DNA analytes concurrently. This approach is referred to as spectral multiplexing.

Unlike a typical fluorophore that emits light at longer wavelengths upon optical excitation, the BHQs or dark quenchers absorb excitation energy but dissipate the energy of excitation via vibrational relaxation, hence have no associated emission spectra [23]. The absorption and emission spectra of the two FRET pairs used in this work, and the corresponding spectral overlap is shown in Fig. 3. Fig. 3(a) and (b) depict gQDs donor with BHQ1-labeled acceptors and rQDs donor with BHQ2-labeled acceptors, respectively. The spectral overlap for the two FRET systems is denoted by the shaded area. The spectral overlap integrals for the gQDs/BHQ1 (donor/acceptor) and rQDs/BHQ2 (donor/acceptor) FRET pairs are $3.2 \times 10^{-10} \mathrm{~cm}^{6} \mathrm{~mol}^{-1}$ and $5.9 \times 10^{-10} \mathrm{~cm}^{6} \mathrm{~mol}^{-1}$, respectively.

\section{Fluorescent Contact Imaging Microsystem}

To enable small-form-factor, point-of-care DNA analysis, a fluorescent contact imaging microsystem can be used to excite the fluorescent markers and quantitatively detect their emission, which is representative of the target concentration. In order to aid microsystem design optimization, a quantitative model of the fluorescent excitation, emission, and detection process is first presented, followed by a description of a microsystem prototype.

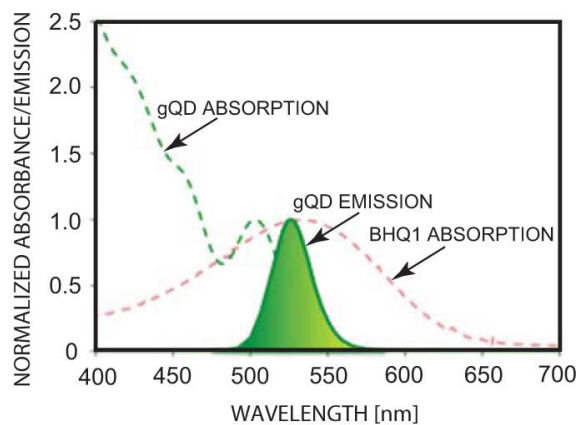

(a)

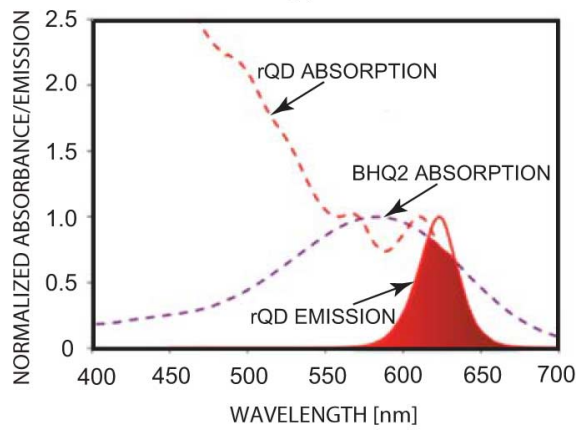

(b)

Fig. 3. Measured absorption and emission spectra of the two FRET pairs used in this work (a) green QDs donor with BHQ1-labeled acceptors (peak AB = $534 \mathrm{~nm}$ ) and (b) red QDs donor with BHQ2-labeled acceptors (peak AB = $583 \mathrm{~nm}$ ). The spectral overlaps are shown as the shaded area.

\section{A. Microsystem Model}

A physical model has been developed for the fluorescent contact imaging microsystem shown in Fig. 1. The microsystem consists of a light-emitting diode (LED) for excitation, an optical filter for excitation light rejection, a fluidic structure to hold the sample solution, and a sensor for photo detection. The sample is assumed to be a solution containing fluorescent molecules, e.g., fluorophores. The formulation seeks to estimate the amplitude and signal-to-noise ratio (SNR) of the fluorescence emission light that is measured by the photo sensor outputting a digital signal. Beginning the analysis with the excitation light, the Beer-Lambert Law can be used to describe the absorption of light traveling through a medium, specifically

$$
T=\frac{I}{I_{0}}=10^{-\epsilon c_{f} l}
$$

where $T$ is the transmissivity or fraction of the excitation light that travels through the sample medium, in this case a layer of fluorophore. It is defined as the ratio of the transmitted excitation light $I$ to the intensities of the incident excitation light $I_{0} . \epsilon$ is the molar absorptivity of a fluorophore, $c_{f}$ is the fluorophore molar concentration, and $l$ is the length of the light path through the medium, i.e., the thickness of the medium, given by

$$
l=\frac{V_{f}}{A_{f}}
$$


where $V_{f}$ and $A_{f}$ are the fluorophore sample solution volume and the fluorophore footprint area that the solution occupies, respectively.

The absorptivity or the fraction of the excitation light absorbed by the fluorophores can be expressed as

$$
F_{a}=1-T
$$

The excitation photon rate, $R_{e x}$, is the rate at which excitation photons are successfully absorbed by the fluorophore sample, given by

$$
R_{e x}=F_{a} \frac{P_{e x}}{E_{p h}}
$$

where $P_{e x}$ is the power of the excitation light that falls onto the fluorophore sample area $A_{f}$, given by

$$
P_{e x}=P_{s r c} \frac{A_{f}}{A_{s r c}},
$$

where $P_{s r c}$ and $A_{s r c}$ are the power and the total illuminated area of the excitation source, respectively. $E_{p h}$ is the photon energy of the excitation light, given by

$$
E_{p h}=\frac{h c}{\lambda_{e x}}
$$

where $h$ is Planck's constant, $c$ is the speed of light and $\lambda_{e x}$ is the wavelength of the excitation light.

The rate of the fluorophore emission light that reaches the photo sensor pixel area can be estimated by

$$
R_{e m}=\eta_{c p} \eta_{f i l t e r} \eta_{d y e} R_{e x}
$$

where $\eta_{c p}$ is the fluorescent emission collection efficiency of the pixel, $\eta_{\text {filter }}$ is the filter transmissivity at the emission light wavelength, and $\eta_{d y e}$ is the quantum yield of the fluorophore. $\eta_{c p}$ is given by

$$
\eta_{c p}=\eta_{c p}^{\prime} A_{p i x}
$$

where $\eta_{c p}^{\prime}$ is the fluorescent emission collection efficiency per unit area and $A_{p i x}$ is the pixel area.

The photodetector current can be expressed as

$$
I_{p d}=q k_{p d} \eta_{f f} R_{e m}
$$

where $q$ is the electron charge, $k_{p d}$ is the responsivity of the photodetector which is temperature-dependent, and $\eta_{f f}$ is the fill factor of the sensor pixel. The voltage developed at the end of the integration time $T_{i n t}$ at the output of a direct-integration photodetector can be expressed as

$$
V_{p d}=\frac{I_{p d} T_{i n t}}{C_{p d}}
$$

where $C_{p d}$ is the pixel integration capacitance, often implemented by the photodetector parasitic capacitance.

The voltage $V_{p d}$ is often digitized by an ADC. For a self-reset based current-to-frequency ADC [17], the digital output is given by

$$
D_{\text {out }}=\frac{V_{p d}}{V_{\text {ref }}-V_{r s t}}
$$

where $V_{r e f}$ and $V_{r s t}$ are the reference voltage and reset voltage, respectively. Finally, the SNR of the emission signal is given by

$$
S N R(d B)=20 \log \left(\frac{D_{\text {out }}}{\sigma_{\text {out }}}\right)
$$

where $\sigma_{\text {out }}$ is the standard deviation of $D_{\text {out }}$ at the background level (i.e., when $R_{e m}=0$ ). This standard definition of SNR assumes noise is independent of the input amplitude. This assumption is valid for a read noise dominate case, for example, an imager fabricated in a standard CMOS technology sensing a fluorescence signal typically of a low intensity [5], [24]. $\sigma_{\text {out }}^{2}$ is the noise power, which is given by

$$
\sigma_{\text {out }}^{2}=\sigma_{e}^{2}+\sigma_{n e}^{2}
$$

where $\sigma_{e}^{2}$ and $\sigma_{n e}^{2}$ are the noise power components attributed to sensor electronics and non-electronic mechanisms, respectively. $\sigma_{e}^{2}$ consists of noise sources such as detector dark noise, readout circuit thermal and flicker noise, and ADC quantization noise. Noise associated with other non-electronic mechanisms of the microsystem includes various well-studied effects such as randomness in excitation scattering [25], autofluorescence [26], and non-specific absorption [27]. The detection limit is conventionally defined [28] as the signal that is equal to $3 \sigma_{\text {out }}$, i.e., the signal at which $\mathrm{SNR}=3(9.54 \mathrm{~dB})$.

To summarize, (2), (5), and (7) are associated with the chemistry and optics of the microsystem, whereas (9)-(11) are associated with VLSI circuit implementation. Equation (11) and (12) are utilized to estimate the amplitude and SNR of the observed fluorescence signal as it relates to various microsystem parameters.

For the measurement of noise parameters, $\sigma_{e}^{2}$ can be obtained by directly illuminating the sensor pixel by a controlled light source whose intensity is calibrated to the level that produces $D_{\text {out }}$. The output noise $\sigma_{\text {out }}$ can be obtained by using a non-fluorescent buffer solution to represent the zero signal (i.e., $R_{e m}=0$ ) and captures the effects of non-idealities in the microsystem. Noise associated with other non-electronic mechanisms of the microsystem can be obtained by de-embedding the electronic noise from the output noise, i.e., $\sigma_{n e}^{2}=\sigma_{o u t^{\prime}}^{2}-\sigma_{e}^{2}$.

\section{Microsystem Prototype}

Fig. 4 is a photograph of the prototyped microsystem. The microsystem consists of a blue LED for excitation, a collimator to ensure the excitation light reaching the sample and the filter has a normal angle of incidence, an optical emission filter, a fluidic structure for holding the sample solution, and the CMOS CPG sensor for photo detection. 


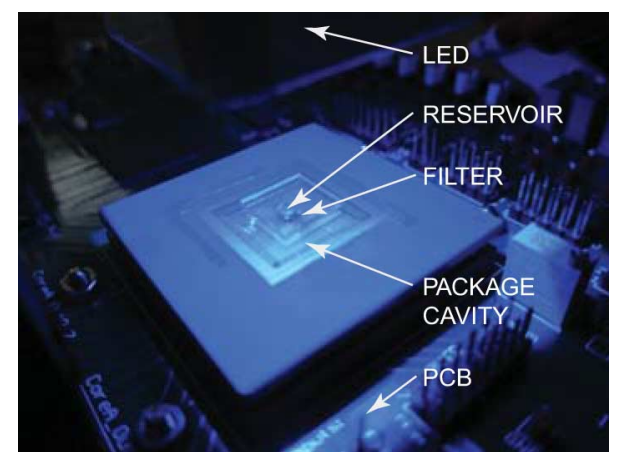

Fig. 4. Fluorescence detection microsystem prototype.

\section{A. Filter}

The optical filter chosen for the microsystem is a discrete thin-film interference filter for attenuating the excitation light from the Philips Luxeon K2 $450 \mathrm{~nm}$ blue LED excitation $(\mathrm{FWHM}=20 \mathrm{~nm})$ [29]. It has been designed, fabricated (Omega Optical) [30], and optically tested prior to integration with the CMOS die. This approach is chosen over the direct deposition of thin-film layers over the CMOS die to ensure that proven methods for coating planar substrates can be used during filter fabrication. Direct thin-film deposition over the CMOS die involves higher costs due to complications in adjusting the fabrication process to compensate for the temperature and material differences between the surface of the optical filter and the CMOS die. The encapsulation of bond wires for protection during the coating process in the direct filter deposition method requires additional costs.

In the proposed microsystem, a $100 \mu$ m-thick, $2.5 \mathrm{~cm} \times$ $2.5 \mathrm{~cm}$ optical filter is diced into several smaller $1.5 \mathrm{~mm} \times$ $1.5 \mathrm{~mm}$ pieces to match the size of the CMOS pixel array. Fabricating multiple filters by dicing a single filter significantly reduces the unit cost. The interference filter is fabricated using 60 layers of $\mathrm{Nb}_{2} \mathrm{O}_{5}$ and $\mathrm{SiO}_{2}$. These materials are selected for their durability and optical properties. The coatings are deposited onto a $100 \mu \mathrm{m}$-thick microsheet of fused silica substrate by vapor deposition. The coated substrate is then cut with a diamond saw to the size required to cover the pixel array. Finally, the diced filter is attached to the CMOS die.

The long-pass interference filter has a cut-off wavelength of $510 \pm 2 \mathrm{~nm}$. The cut-off wavelength is chosen to transmit the emitted light from the gQDs and rQDs, with the peak emission wavelengths of $520 \mathrm{~nm}$ and $620 \mathrm{~nm}$, respectively. The transmission of the filter is greater than $90 \%$ at $520 \mathrm{~nm}$ and on average greater than $85 \%$ from $520 \mathrm{~nm}$ to $700 \mathrm{~nm}$, as depicted in Fig. 5 . The filter has been tested under laboratory conditions to provide an optical density (OD) of six (e.g., $10^{6}$ attenuation) at the excitation wavelength of $450 \mathrm{~nm}$ for an angle of incidence of up to 20 degrees from the normal.

\section{B. Fluidic Structure}

To ameliorate the scattering effects caused by uneven drying of the samples, the QDs are imaged in the solution phase as opposed to solid phase. For a tight control of the geometry of the QD solution to be sensed, a micro-reservoir is fabricated to

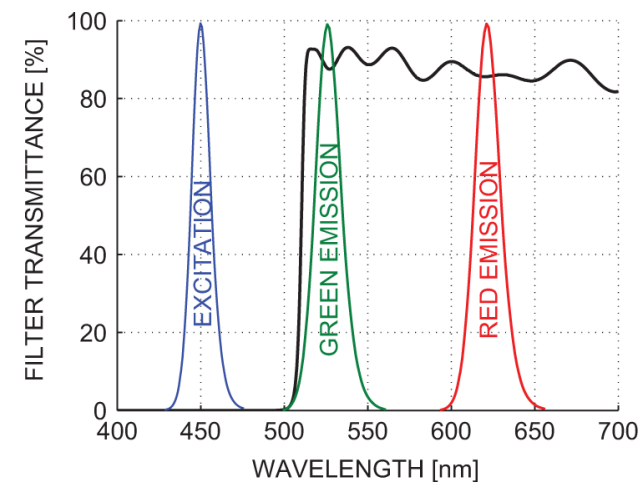

Fig. 5. Measured thin-film filter transmission characteristics.

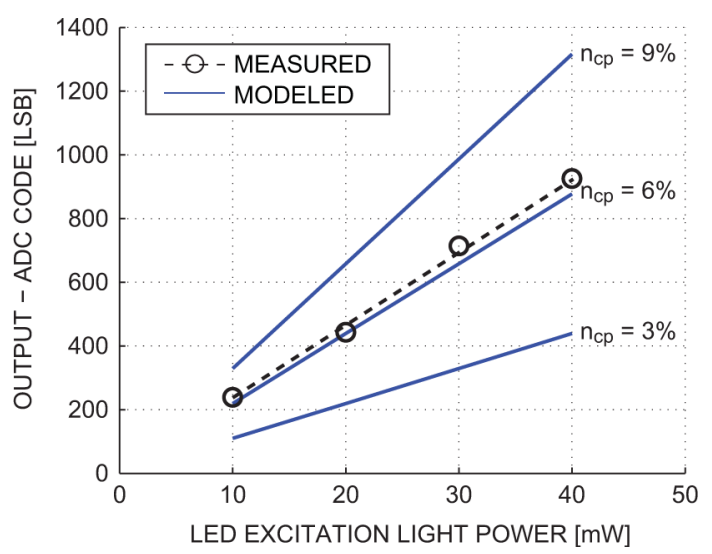

Fig. 6. Sensor digital output for $10 \mu \mathrm{L}$ of $2 \mu \mathrm{M}$ red $(620 \mathrm{~nm}) \mathrm{QD}$.

contain the solution instead of depositing the QD on a surface, such as a glass slide.

To fabricate the reservoir, a circular volume is removed by a metal punch from a piece of polydimethylsiloxane (PDMS), which is subsequently plasma bonded to a glass cover slip. The dimensions of the cover slip, which forms the bottom of the reservoir, are $22 \mathrm{~mm}$ (length) $\times 22 \mathrm{~mm}$ (width) $\times 150 \mu \mathrm{m}$ (thickness). The bottom of the reservoir is made as thin as possible to minimize the distance between the sample and the focal plane. The $4 \mathrm{~mm}$-diameter reservoir, with a side wall height of $1 \mathrm{~mm}$, holds a sample volume of approximately $10 \mu \mathrm{L}$.

\section{Microsystem Model Validation}

As a form of validation, the physical model is compared with experimental results. In particular, the model has been used to describe the effect of varying the excitation power on the sensor output of the prototype depicted in Fig. 4. Fig. 6 depicts the sensor output from measuring the emission of $10 \mu \mathrm{L}$ of $2 \mu \mathrm{M}$ red $(620 \mathrm{~nm})$ QD solution. The values of model parameters used are enumerated in Table I. Four measurements are performed at $10 \mathrm{~mW}, 20 \mathrm{~mW}, 30 \mathrm{~mW}$, and $40 \mathrm{~mW}$ of blue LED excitation power. Sensor output has been calculated for several pixel collection efficiencies $\eta_{c p}$ and from correlating the model with measurements, it is found that $\eta_{c p}$ is approximately $6 \%$.

\section{CMOS Color-Photogate Sensor}

The complexity of the microsystem is significantly reduced by the integration of a CMOS color sensor, which is based on 
TABLE I

Microsystem Model Parameters and Physical Constants

\begin{tabular}{|c|c|c|c|}
\hline Symbol & Model Parameter & Value & Unit \\
\hline$\epsilon$ & $\begin{array}{l}\text { Primary Design Parameters } \\
\text { molar absorptivity of fluorophore at } \lambda_{e x}\end{array}$ & $1.00 \times 10^{8}$ & $\mathrm{M}^{-1} \mathrm{~m}^{-1}$ \\
\hline$V_{f}$ & fluorophore sample volume & $1 \times 10^{-8}$ & $\mathrm{~m}^{3}$ \\
\hline$A_{f}$ & fluorophore sample area & $4 \times 10^{-6}$ & $\mathrm{~m}^{2}$ \\
\hline$c_{f}$ & fluorophore molar concentration & $2 \times 10^{-6}$ & $\mathrm{M}=\mathrm{mol} / \mathrm{L}$ \\
\hline$P_{s r c}$ & power of the excitation source & $40 \times 10^{-3}$ & $\mathrm{~W}$ \\
\hline$A_{s r c}$ & illuminated area of the excitation source & $4 \times 10^{-4}$ & $\mathrm{~m}^{2}$ \\
\hline$\lambda_{e x}$ & excitation wavelength & $450 \times 10^{-9}$ & $\mathrm{~m}$ \\
\hline$\eta_{c p}$ & fluorescent emission collection efficiency of pixel & 0.06 & - \\
\hline$\eta_{c p}^{\prime}$ & $\eta_{c p}$ per unit area & $1.96 \times 10^{6}$ & $\mathrm{~m}^{-2}$ \\
\hline$A_{p i x}$ & pixel area & $3.06 \times 10^{-8}$ & $\mathrm{~m}^{2}$ \\
\hline$\eta_{\text {filter }}$ & filter transmissivity at emission wavelength & 0.85 & - \\
\hline$\eta_{\text {dye }}$ & fluorophore quantum yield & 0.25 & - \\
\hline$k_{p d}$ & photodetector responsivity & 0.30 & $\mathrm{~A} / \mathrm{W}$ \\
\hline$\eta_{f f}$ & sensor pixel fill factor & 0.10 & - \\
\hline$T_{\text {int }}$ & integration time & 1.00 & $\mathrm{~s}$ \\
\hline$C_{p d}$ & pixel integration capacitance & $500 \times 10^{-15}$ & $\mathbf{F}$ \\
\hline$V_{\text {ref }}$ & ADC reference voltage & 0.10 & V \\
\hline$V_{\text {rst }}$ & pixel reset voltage & 0 & $\mathrm{~V}$ \\
\hline$\sigma_{e}$ & standard deviation of electronic noise & 3.39 & LSB \\
\hline \multirow[t]{2}{*}{$\sigma_{n e}$} & standard deviation of non-electronic noise & 7.72 & LSB \\
\hline & Intermediate Design Parameters & & \\
\hline$T$ & light transmissivity through fluorophore & 0.74 & - \\
\hline$l$ & optical path length through fluorophore & $2.50 \times 10^{-3}$ & $\mathrm{~m}$ \\
\hline$F_{a}$ & absorptivity of excitation by fluorophores & 0.26 & - \\
\hline$R_{e x}$ & excitation photon rate & $3.24 \times 10^{14}$ & photons/s \\
\hline$P_{e x}$ & excitation power illuminating fluorophore area & $4.00 \times 10^{-4}$ & $\mathrm{~W}$ \\
\hline$E_{p h}$ & energy per excitation photon & $3.21 \times 10^{-19}$ & $\mathrm{~J}$ \\
\hline$R_{e m}$ & rate of fluorophore emission collected by pixel & $9.14 \times 10^{9}$ & photons/s \\
\hline$I_{p d}$ & photodetector current & $4.39 \times 10^{-11}$ & $\mathrm{~A}$ \\
\hline$V_{p d}$ & photodetector output voltage & 88.7 (effective) & $\mathrm{V}$ \\
\hline$D_{\text {out }}$ & ADC digital output & $887\left(P_{s r c}=40 \mathrm{~mW}\right)$ & LSB \\
\hline & standard deviation of ADC digital output & 9.26 (at no fluores.) & LSB \\
\hline \multirow[t]{2}{*}{$S N R$} & signal-to-noise ratio of microsystem & $39.6\left(P_{s r c}=40 \mathrm{~mW}\right)$ & $\mathrm{dB}$ \\
\hline & Physical Constants & & \\
\hline$N_{A}$ & Avogadro's number & $6.02 \times 10^{23}$ & molecules $/ \mathrm{mol}$ \\
\hline$h$ & Planck's constant & $6.63 \times 10^{-34}$ & $\mathrm{~J} \mathrm{~s}$ \\
\hline$c$ & speed of light & $3.00 \times 10^{8}$ & $\mathrm{~m} / \mathrm{s}$ \\
\hline$q$ & electron charge & $1.60 \times 10^{-19}$ & coulomb \\
\hline
\end{tabular}

the color photogate (CPG). The CPG spectrally detects and differentiates the multi-color emission, eliminating the need to mechanically swapped filters. The CPG further performs analog-to-digital conversion.

\section{A. Principle of Operation}

When multiple wavelengths of light are incident simultaneously, the intensities at these wavelengths can be determined by measurements from several photo detectors with unique spectral responses. For example, for a two-wavelength input, the photo currents $I_{1}$ and $I_{2}$ measured by two photo detectors can be related to the input intensities $\phi_{1}$ and $\phi_{2}$, at wavelengths $\lambda_{1}$ and $\lambda_{2}$ respectively, by

$$
\begin{aligned}
& I_{1}=k_{11} \phi_{1}+k_{12} \phi_{2} \\
& I_{2}=k_{21} \phi_{1}+k_{22} \phi_{2}
\end{aligned}
$$

where the $k$-coefficients capture the responsivity of the detectors at different wavelengths and can be obtained empirically. The input intensities $\phi_{1}$ and $\phi_{2}$ can be obtained by solving the system of equations, provided that the detectors have unique spectral responses (i.e., equations are linearly independent). This model can be extended to a finite set of $N$ wavelengths. To determine the intensity of an input spectrum to a resolution of $N$ distinct wavelengths, $N$ measurements are required from the $N$ detectors (one measurement per detector), with each detector having a unique spectral response.

\section{B. VLSI Implementation}

To create the equivalent of multiple photo detectors with unique spectral responses, the CPG depicted in Fig. 7 has been utilized [17]. It is comprised of the core and edge regions. The core region of the CPG is covered by a polysilicon gate. A $p^{+}$diffusion, referred to as the edge region, forms the device output. An $n^{+}$diffusion fabricated in an $n$-body forms the body bias ohmic contact. The entire CPG is physically implemented in an $n$-well for isolation against the substrate noise and, potentially, crosstalk from adjacent pixels in a sensor array. The $p^{+}$output diffusion is limited by readout circuit operation to a voltage lower than the $n$-well voltage to ensure a reverse biased junction. The $\mathrm{CPG}$ has a structural resemblance to the 


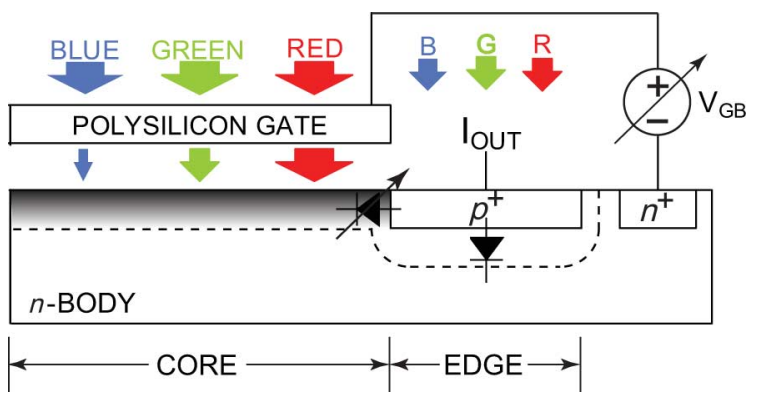

Fig. 7. Cross-sectional view of the CMOS color photogate (CPG).

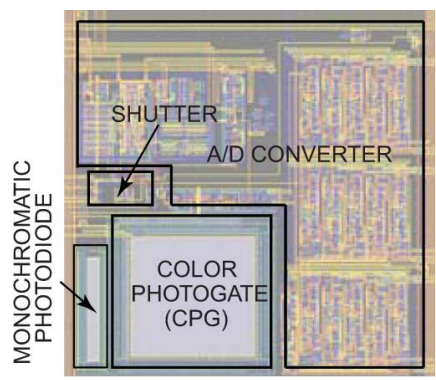

(a)

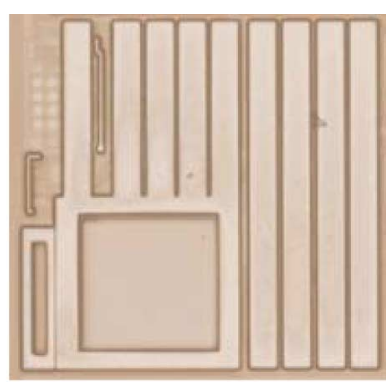

(b)
Fig. 8. $175 \mu \mathrm{m} \times 175 \mu \mathrm{m}$ pixel with a $50 \mu \mathrm{m} \times 50 \mu \mathrm{m} \mathrm{CPG}$ in a $0.35 \mu \mathrm{m}$ standard CMOS technology: (a) layout and (b) micrograph.

conventional CMOS monochromatic $p^{+} / n$-body photodiode and is an integrated approach to coarse color differentiation for multi-color fluorescence detection.

The gate performs two key functions. First, it is a terminal for the application of the electric field to modulate the efficiency of photo-generated carrier collection in the core region, the area under the gate. As the gate-to-body voltage $V_{G B}$ changes, the CPG transits through modes of operations, analogous to a metaloxide-semiconductor (MOS) capacitor. This mode change leads to a change in the carrier collection efficiency, which ultimately leads to a change in the device photocurrent.

When a high $V_{G B}$ is applied so that no depletion region is formed under the MOSFET gate, photocurrent generation only takes place at the $p^{+} / n$-body depletion region. For a low $V_{G B}$, majority carrier electrons in the $n$-body are repelled from the silicon surface thereby forming a depletion region under the gate, which participates in photo sensing.

The gate also acts as an optical filter. Due to the optical property of polysilicon, light experiences wavelength-dependent absorption as it travels through the gate, as illustrated in Fig. 7. Since absorption is such that the gate provides greater attenuation at shorter wavelengths, the CPG core region is responsible for sensing longer (e.g., red) wavelengths. Since the core and edge regions of the CPG have different spectral properties, by using multiple gate-to-body voltages to activate the core region to different extents, the equivalent of multiple detectors with different spectral responses can be created, implementing (14) and (15) for two gate-to-body voltages in a single device.

The CPG has been prototyped in a $0.35 \mu \mathrm{m}$ standard CMOS technology, as depicted in Fig. 8. Each $175 \mu \mathrm{m} \times 175 \mu \mathrm{m}$ pixel can be readily tiled to form an array for imaging. It consists a $50 \mu \mathrm{m} \times 50 \mu \mathrm{m} \mathrm{CPG}$ and an ADC for digital readout. The

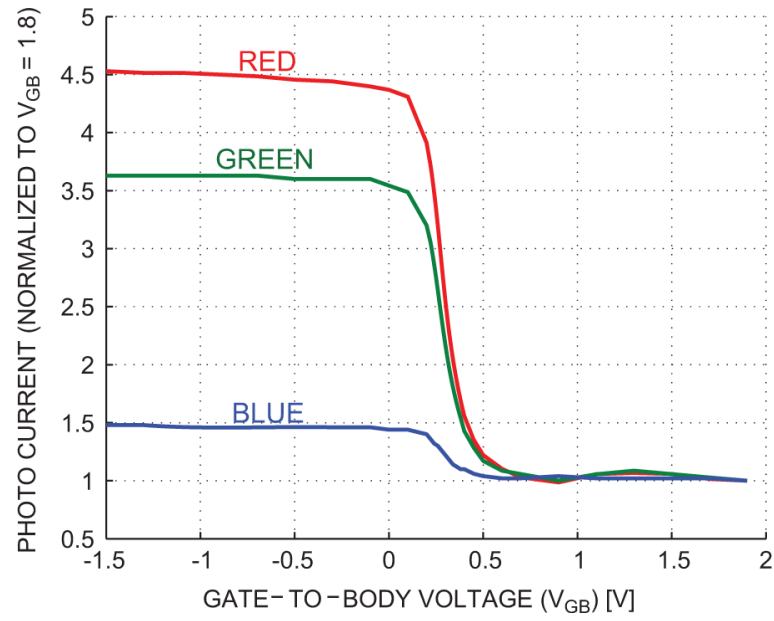

Fig. 9. Measured CPG photocurrent for monochromatic light at $620 \mathrm{~nm}$ (red), $520 \mathrm{~nm}$ (green), and $450 \mathrm{~nm}$ (blue).

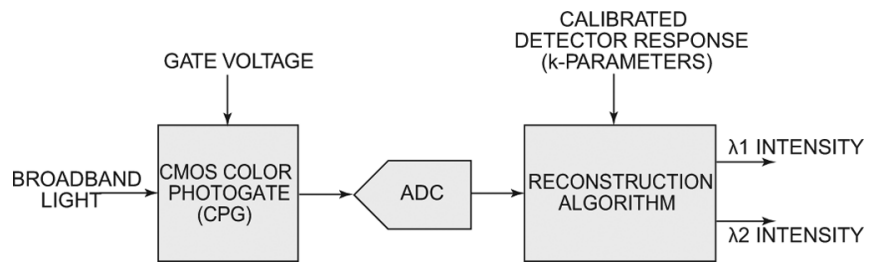

Fig. 10. System diagram of the CMOS color photogate (CPG) sensor.

CPG photocurrent measured across $V_{G B}$ for three monochromatic illuminations using a semiconductor parameter analyser is depicted in Fig. 9. For $0 V<V_{G B}<0.6 \mathrm{~V}$, the ratio of the currents corresponding to each color changes significantly as a function of $V_{G B}$. Therefore, gate voltages within this range are used for the multiple measurements as required in (14) and (15).

Fig. 10 depicts the overall CPG-based sensor architecture. To resolve $N$ colors, $N$ CPG responses at $N$ different values of $V_{G B}$ are passed through the ADC for digitization. The reconstruction algorithm that solves for the input spectrum $\phi$ in (14) and (15) is implemented in software. LED based experimental validation of the CPG design has been reported in [17]. Here we present system design validation in DNA detection applications.

\section{System Validation in DNA Detection}

The microsystem has been verified through both single-target (single-color) DNA detection and simultaneous multi-target (spectrally-multiplexed) DNA detection. For a two-color system, as can be seen in Fig. 9, pairing red QDs with blue instead of green QDs would produce greater linear-independence in the $k$-coefficients in (14) and (15). However, since the blue QD requires ultraviolet excitation light, it is not a widely-adopted fluorescent molecule. Therefore, green and red QDs are chosen to demonstrate the microsystem.

\section{A. Preparation of Quantum Dots}

Oleic acid capped organic core/shell $\mathrm{CdSeS} / \mathrm{ZnS}$ based QDs from Cytodiagnostics [31] have been made water-soluble by a ligand exchange reaction with 3-mercaptopropionic acid [32]. The quantum yields of the mercaptopropionic acid 


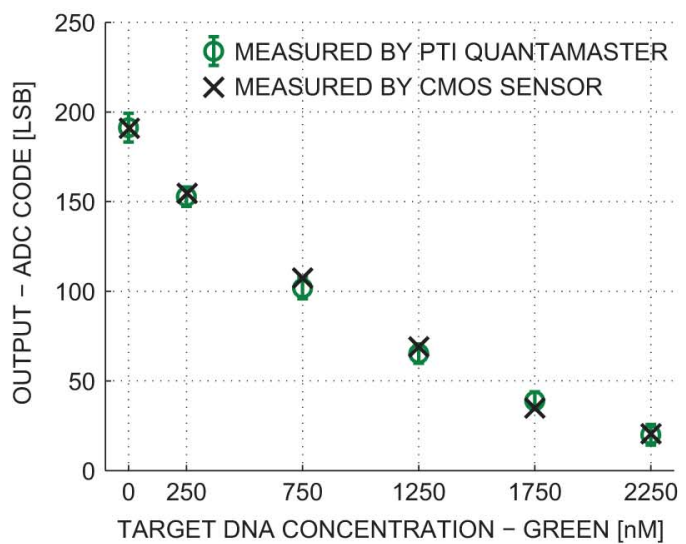

(a)

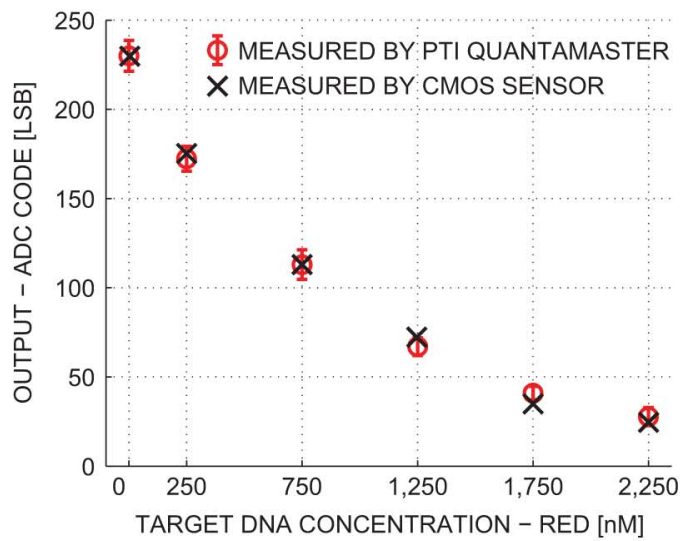

(c)

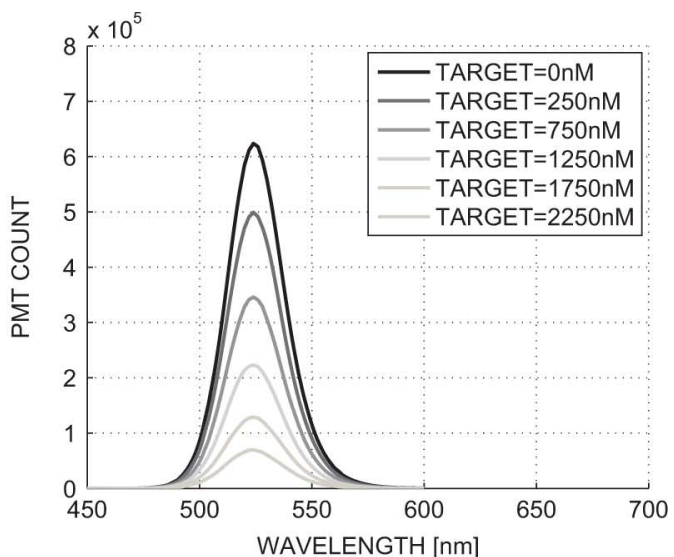

(b)

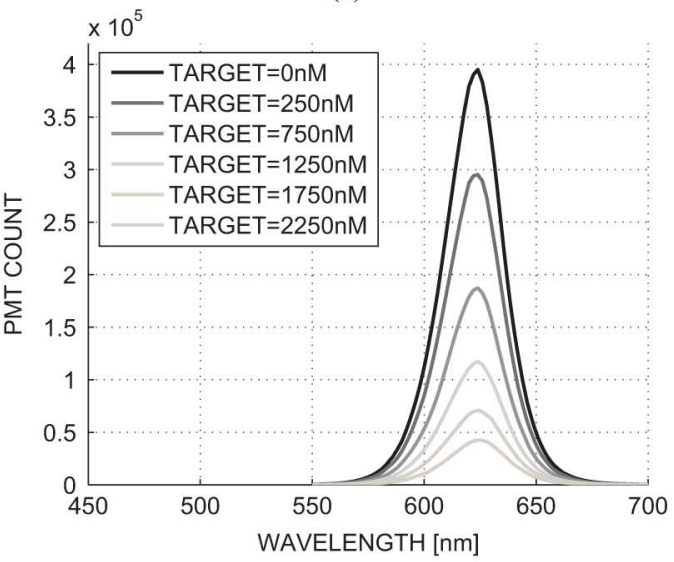

(d)

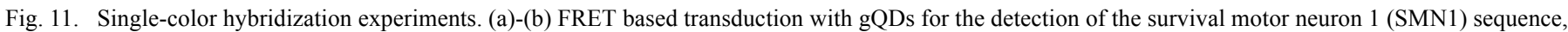

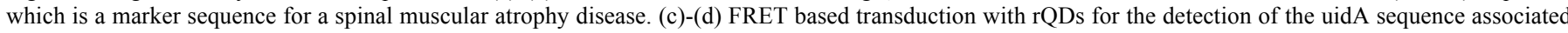

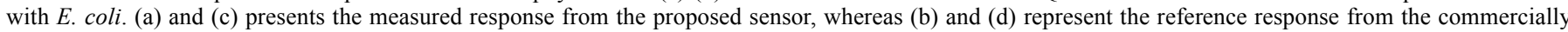

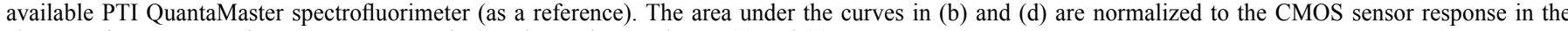
absence of target DNA (i.e., 0 nM concentration) and superimposed onto (a) and (c).

capped gQDs and rQDs are $0.19( \pm 0.02)$ and $0.25( \pm 0.03)$, respectively. The peak emission of gQDs and rQDs are at $520 \mathrm{~nm}$ and $620 \mathrm{~nm}$, respectively, with FWHM $=29 \mathrm{~nm}$ for both colors of QDs. The molar absorptivity $\epsilon$ of quantum dots has been measured by absorption spectroscopy using the HP8452A diode-array spectrophotometer, with the values of $5.90 \times 10^{7} \mathrm{M}^{-1} \mathrm{~m}^{-1}$ and $1.00 \times 10^{8} \mathrm{M}^{-1} \mathrm{~m}^{-1}$ at $450 \mathrm{~nm}$ for gQDs and rQDs, respectively, which are typical for CdSe QDs [33].

\section{B. DNA Hybridization Assays}

As depicted in Fig. 2, to produce a FRET-based fluorescent light output, QDs are conjugated with the DNA probes and DNA targets are labeled with BHQs. The SMN1 and uidA sequences, which are marker sequences for a spinal muscular atrophy disease and for $E$. coli respectively, are utilized as DNA targets. For experiments involving single-color QD solutions, $250 \mathrm{nM}$ of gQD-SMN1 probe conjugates are incubated with $0 \mathrm{nM}, 250 \mathrm{nM}$, $750 \mathrm{nM}, 1250 \mathrm{nM}, 1750 \mathrm{nM}$ and $2250 \mathrm{nM}$ concentrations of BHQ1-labeled SMN1 targets. The hybridization has been done overnight in $50 \mathrm{mM}$ borate buffer with $250 \mathrm{mM} \mathrm{NaCl}$ at pH9.25. Similarly, $250 \mathrm{nM}$ of rQD-uidA probe conjugates are incubated with various aforementioned concentrations of BHQ2-labeled uidA targets.

For the multi-color experiments, gQD-SMN1 probe conjugates and rQD-uidA probe conjugates at $250 \mathrm{nM}$ concentrations each have been incubated with $(0 \mathrm{nM}, 0 \mathrm{nM}),(250 \mathrm{nM}, 250 \mathrm{nM})$, $(1250 \mathrm{nM}, 1250 \mathrm{nM}),(2250 \mathrm{nM}, 2250 \mathrm{nM}),(250 \mathrm{nM}, 2250 \mathrm{nM})$ and $(2250 \mathrm{nM}, 250 \mathrm{nM})$ concentrations of BHQ-targets. The first number in the parentheses corresponds to the concentration of BHQ1-labeled SMN1 target, whereas the second number corresponds to the concentration of BHQ2-labeled uidA target. The hybridization condition is kept the same as that of single-color hybridization experiments.

\section{Single-Target DNA Detection}

Fig. 11 shows single-color hybridization experiments with both colors of QDs demonstrating the applicability of the assay and the color photogate to quantitatively detect BHQ-labeled DNA targets via FRET-based quenching of QD emission intensity. A hybridization event between the immobilized probes on the surface of QD and BHQ labeled targets provided proximity necessary for FRET-based energy transfer between the donors (gQDs and rQDs) and the acceptors (BHQ1 and BHQ2). Fig. 11(a) and (b) depict measurements from a $250 \mathrm{nM}$ solution 
of gQD-SMN1 probe conjugates, with $0 \mathrm{nM}, 250 \mathrm{nM}, 750 \mathrm{nM}$, $1250 \mathrm{nM}, 1750 \mathrm{nM}$, and $2250 \mathrm{nM}$ of BHQ1-labeled SMN1 targets. Fig. 11(c) and (d) depict measurements from a $250 \mathrm{nM}$ solution of rQD-uidA probe conjugates with $0 \mathrm{nM}, 250 \mathrm{nM}$, $750 \mathrm{nM}, 1250 \mathrm{nM}, 1750 \mathrm{nM}$, and $2250 \mathrm{nM}$ of BHQ2-labeled uidA targets.

Fig. 11(a) and (c) depict intensity measurements from the proposed CPG sensor for various DNA target concentrations, for a 1 second integration time. A total of eight measurements were taken for a total integration time of 8 seconds. The error bars show one standard deviation of the variation across the eight measurements. As a reference, Fig. 11(b) and (d) depict QD emission intensities measured by the commercially available QuantaMaster PTI spectrofluorimeter in the presence of a filter, with an optical density of 1 (i.e., $10 \times$ attenuation), to attenuate the excitation. For comparison, the area under the curves in Fig. 11(b) and (d) are normalized to the CMOS sensor response in the absence of target DNA (i.e., $0 \mathrm{nM}$ concentration) and superimposed onto Fig. 11(a) and (c).

Since there is only one emission band in single-color experiments, emission intensity is the only quantity to be measured as the wavelength is known. As a result, for single-color experiments, the CPG sensor functions as a regular monochromatic sensor, i.e., mathematical reconstruction is not necessary.

Fig. 11 shows that the measurements made using the CPG sensor correlate well with the measurements made using the commercial spectrofluorimeter. For both QD emission bands, the intensity has been observed to decrease with increasing target concentration. This is as expected since increasing the target concentration allowed for a greater number of acceptors (BHQs) to interact with the donors (QDs), resulting in an increase in FRET-based energy transfer, hence a decrease in the QD emission intensity.

The results of Fig. 11 exhibit the expected behavior of exponential reduction in the fluorescence emission intensity of QDs with increasing concentrations of targets. The exponential nature of the response with increasing target concentrations is due to the exponential changes in FRET efficiency with increasing number of acceptors, i.e., the target concentration [34].

It is worth noting that since the signal transduction is based on FRET, the quenching of the QD emission intensity only occurs upon the hybridization of targets with probes on the QD surface. As a result, a washing step requiring removal of excess non-hybridized target from the solution is not necessary, which provides an opportunity for real-time detection of hybridization profiles.

It can also be observed from Fig. 11(a) and (c) that when the DNA targets are not present (i.e., target concentration is zero), sensor output signals of 200 codes $(\sigma=8.0)$ and 230 codes $(\sigma=8.6)$ are present for the green and red transduction channels, respectively. Based on the conventional $3 \sigma$ definition, the detection limits of $170 \mathrm{nM}$ and $80 \mathrm{nM}$ are achieved for the targets associated with green and red channels, respectively. It is worth noting that the ratio of these detection limits can be predicted by the model developed in Section III as the molar absorptivity of $\mathrm{rQD}$ is approximately $2 \times$ that of the $\mathrm{gQD}$ at the $450 \mathrm{~nm}$ excitation wavelength. Also, since $10 \mu \mathrm{L}$ of sample volume is used for each measurement with detection limits ex-

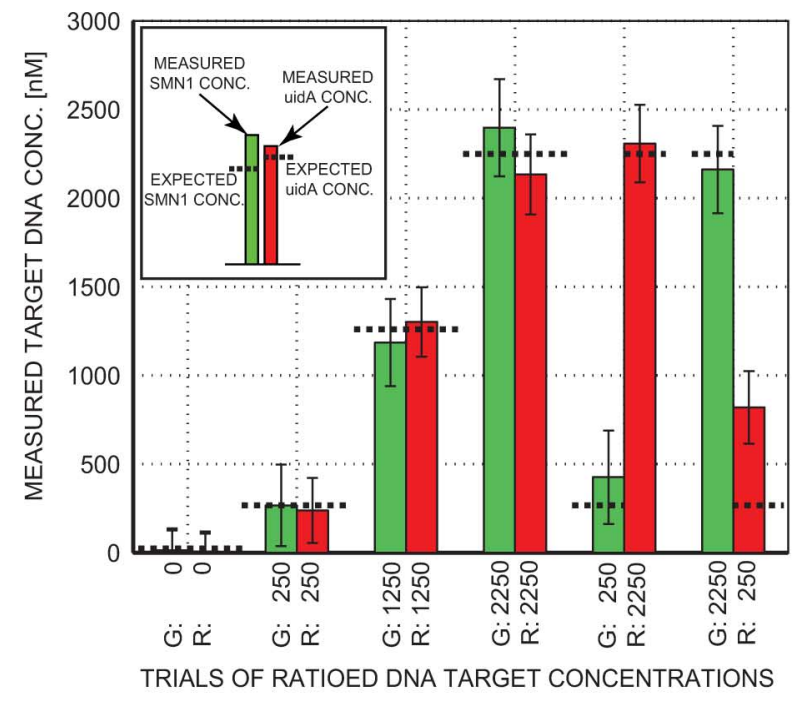

(a)

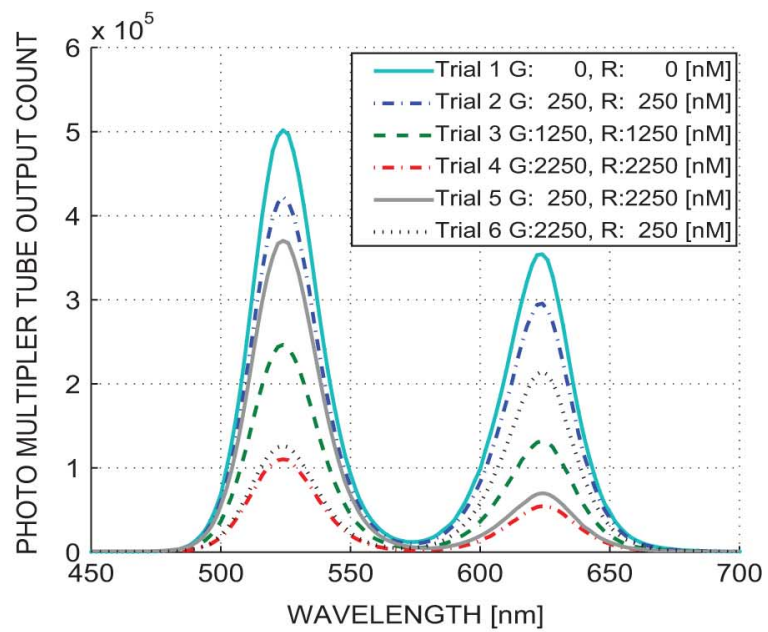

(b)

Fig. 12. Spectrally-multiplexed simultaneous two-target DNA detection. The survival motor neuron 1 (SMN1) sequence is a marker for a spinal muscular atrophy disease and the uidA sequence is a marker for E. coli (a) measured with the proposed sensor, and (b) measured with a spectrofluorimeter (as a reference).

pressed in terms of concentration of $170 \mathrm{nM}$ and $80 \mathrm{nM}$, the microsystem is able to detect $1.7 \mathrm{pmol}$ and $0.8 \mathrm{pmol}$ of target DNA for the green and red channels, respectively.

\section{Simultaneous Two-Target DNA Detection}

The single-color green and red QD systems have been spectrally-multiplexed into a multi-color system for the simultaneous detection of SMN1 and uidA targets, with results shown in Fig. 12. Fig. 12 depicts six trials each with different combinations of two DNA target concentrations. The data shown in Fig. 12(a) and (b) are obtained from the DNA bioassays by measuring the response with the proposed sensor and a commercial spectrofluorimeter as a reference, respectively. These trials are two-color experiments demonstrating the ability of the CPG to concurrently detect two different DNA targets via FRET-based quenching of QD emission. Each solution contains gQD-SMN1 probe conjugates and rQD-uidA probe conjugates at $250 \mathrm{nM}$ concentrations each, and are incubated with $(0 \mathrm{nM}, 0 \mathrm{nM}),(250 \mathrm{nM}, 250 \mathrm{nM}),(1250 \mathrm{nM}, 1250 \mathrm{nM})$, 
$(2250 \mathrm{nM}, 2250 \mathrm{nM}),(250 \mathrm{nM}, 2250 \mathrm{nM})$ and $(2250 \mathrm{nM}$, $250 \mathrm{nM}$ ) concentrations of BHQ1-labeled SMN1 targets and BHQ2-labeled uidA targets (denoted as the first and second number in the parentheses, respectively). The integration time for each trial is 1 second.

To invoke the spectral sensing capability of the sensor, the input is sampled at multiple CPG gate-to-body voltages. In this case of detecting two colors of QD emissions, two values of $V_{G B}$ are used. To improve detection accuracy, for each combination of DNA target concentrations, eight measurements are performed at each of the two gate voltages $\left(V_{G B 1}=0 \mathrm{~V}\right.$, $V_{G B 2}=0.6 \mathrm{~V}$, with $V_{B O D Y}=1.5 \mathrm{~V}$ ). The data from each set are then averaged, background subtracted, and fed into the reconstruction algorithm to solve for the input intensities at each of the two wavelength bands that indicate the respective concentrations of two targets in terms of the emission intensities from both colors of QDs.

It is worth noting that the measurements shown in Fig. 12(a) are based on measured QD emission intensities expressed in terms of concentration from the intensity-to-concentration relationships established in Fig. 11(a) and (c) for the green and red QDs based FRET systems, respectively. For example, for the $(0 \mathrm{nM}, 0 \mathrm{nM})$ trial in Fig. 12(a), since the absence of both DNA targets results in no quenching of QD emission in the green and red channels, the CPG sensor output, which measures the QD emission intensity is actually the highest of the six trials.

It has been observed that the QD emission intensity of a certain transduction channel is affected by the degree of quenching in a neighboring channel [e.g., in Fig. 12(b), compare trials 2 and 5 for the green channel, and trials 2 and 6 for the red channel]. This form of crosstalk is attributed to the effects of non-specific absorption [35] in the bioassay, not the CPG sensor. For example, in trial 5, a large degree of quenching in the rQD emission channel due to the presence of high concentration of BHQ2-labeled uidA target also impacts the gQD emission channel. This results in a signal reduction in the gQD emission channel to a greater extent than in trial 2 despite the presence of the same amount of SMN1 targets in both trials. This accounts for the overestimation in trials 5 and 6 of Fig. 12(a). Nonetheless, non-specific absorption can be suppressed by using denatured bovine serum albumin to passivate the surface of QDs [35].

It can be observed from Fig. 12(a) that when the DNA targets are not present (i.e., target concentration is zero), the sensor reports the expected averaged result of approximately zero target concentration with $\sigma \approx 80 \mathrm{nM}$ and $\sigma \approx 70 \mathrm{nM}$ for the green and red transduction channels, respectively. Based on the conventional $3 \sigma$ definition, the detection limits of $240 \mathrm{nM}$ and $210 \mathrm{nM}$ are achieved for the targets associated with green and red channels, respectively. Since $10 \mu \mathrm{L}$ of sample volume is used for each measurement with detection limits in terms of concentration at $240 \mathrm{nM}$ and $210 \mathrm{nM}$, the microsystem is able to detect $2.4 \mathrm{pmol}$ and $2.1 \mathrm{pmol}$ of target DNA for the green and red channels, respectively.

\section{DISCUSSION}

Although the CPG exploits the gate structure in standard CMOS technology, thus requiring no external filter, the poly-gate nonetheless attenuate the incoming light before it is sensed. In the $0.35 \mu \mathrm{m}$ CMOS process, the thickness of the polysilicon gate is approximately $300 \mathrm{~nm}$ [36], leading to an approximate attenuation of $65 \%, 30 \%$, and $15 \%$ for blue $(450 \mathrm{~nm})$, green $(520 \mathrm{~nm})$, and red $(620 \mathrm{~nm})$ light, respectively. This results in a reduction in sensitivity especially in short $(\leq 400 \mathrm{~nm})$ wavelengths. One possible solution is to integrate a standard CMOS photodiode in the pixel, as shown in Fig. 8. The $p^{+} / n$-body photodiode can be used for monochromatic measurements of fluorophores that emits in the blue region, extending the range of fluorophores supported by the microsystem.

In this work, eight samples $(N=8)$ from each $V_{G B}$ have first been averaged, then used in reconstruction. The advantages of averaging $N$ images as opposed to making measurements at $N$ bias voltages can be studied using a two-color example. In the case of averaging, two bias voltages are used. The computations are in taking averages of $N$ samples, followed by solving a system of two equations. In the case of using multiple bias voltages, the question arises concerning the appropiate choices of the $N$ bias voltages. According to Fig. 9, having evenly distributed biases that span across the entire $V_{G B}$ axis would not be a good choice as the use of multiple bias voltages in the 'flat' regions (i.e., $V_{G B}<0 V$ and $V_{G B}>0.6 \mathrm{~V}$ ) would yield poor linear-independence in the $k$-coefficient model. Therefore, the eight samples should be chosen within $0<V_{G B}<0.6 \mathrm{~V}$. In reconstruction, $N$ equations are solved for $N$ unknowns. Assuming that the integration time per sample is much longer than the computation time, as it is likely the case for biological imaging, both cases have approximately the same total integration time. However, the latter case suffers from two problems. First, it has lower scalability to $N$. Fitting $N$ bias voltages within $0<V_{G B}<0.6 \mathrm{~V}$ where $N$ is large would require very precise electronics. Second, in general, the algorithmic complexity is much higher for solving a system of $N$ equations $(N \gg 2)$ than a system of two equations. Therefore, the first approach is preferred.

In conventional microscopes, light penetrates the filter at normal incidence, where interference filters performs well. However, in contact imaging the fluorescence light reaching the detector covers a wide range of angles of incidence because the light emitters are close to the detector. Although experimental results from the prototype have not shown significant background, it is worth noting that excitation rejection can be further improved by filter structures that can tolerate a wide angle of incidence while offering a high optical density, such as a hybrid interference and absorption filter [16].

The range of non-zero target concentrations explored is $250 \mathrm{nM}$ to $2250 \mathrm{nM}$. This corresponds to approximately 1.4 orders of magnitude of dynamic range in terms of detectable DNA target concentration, which is typical of FRET-based systems [20]. Since the nature of the assay involves decrease in QDs fluorescence emission intensity with increasing targets concentration, the $250 \mathrm{nM}$ concentration of QD-probe conjugates have been chosen to provide sufficient initial QD fluorescence emission intensity in the absence of BHQ-labeled targets, to be subsequently quenched in the presence of targets. It is worth noting that the dynamic range of the assay is tunable, depending on the concentration of QD-probe conjugates, as 
previously reported [35]. Hence, target concentrations lower than $250 \mathrm{nM}$ or greater than $2250 \mathrm{nM}$ can be quantitatively determined by simply changing the concentration of QD-probe conjugates. To illustrate, consider the case that nine DNA probe strands are immobilized on a surface of a single QD in a $250 \mathrm{nM}$ QD solution. This system can detect up to a maximum of $2250 \mathrm{nM}$ of target strands, because the total number of probe molecules for $250 \mathrm{nM}$ of QD is $2250 \mathrm{nM}$. Detecting a higher target concentration is not possible because all probes are bound to targets. Therefore, if $125 \mathrm{nM}$ concentration of QD-probe conjugates is used instead of $250 \mathrm{nM}$, the lower limit of detection can be further brought down. However, this also brings down the upper limit of detection, as a smaller number of QD-probe conjugates have a smaller number of probe molecules to hybridize with the targets, as only a certain number of probe molecules can be immobilized on the surface of a QD. On the other hand, if $500 \mathrm{nM}$ concentration of QD-probe conjugates is used instead of $250 \mathrm{nM}$, the upper limit of detection can be extended to $4500 \mathrm{nM}$.

It is often meaningful to characterize the detection limit in terms of the required sample size, instead of solely based on the analyte concentration [37]. Considering the $10 \mu \mathrm{L}$ of sample volume used, the proposed microsystem achieves a detection limit for DNA targets at the picomolar level. This is advantageous especially for applications where the extraction or amplification of sample DNA in large quantities is difficult. As a further benefit, reduction in the sample volume allows for samples to be processed by microfluidic devices, which enables miniaturized and integrated systems.

\section{CONCLUSION}

A spectrally-multiplexed fluorescence detection microsystem for DNA analysis is presented. The microsystem has been quantitatively modeled and prototyped, integrating a CMOS color photogate sensor. The sensor detects and differentiates among the emission light of green and red fluorescent biomarkers without mechanical switching of emission filters. The entire detection system utilizes only one long-pass optical filter for excitation light attenuation. The microsystem has been experimentally verified by the simultaneous detection of two DNA targets with the detection limits of $240 \mathrm{nM}$ and $210 \mathrm{nM}$ for the SMN1 and uidA sequences. The prototype is an enabling technology for miniaturized, low-cost, and parallel gene-based point-of-care diagnostics.

\section{REFERENCES}

[1] X. Gao, Y. Cui, R. Levenson, L. Chung, and S. Nie, "In vivo cancer targeting and imaging with semiconductor quantum dots," Nature Biotechnol., vol. 22, no. 8, pp. 970-976, Jul. 2004.

[2] A. Chagovetz and S. Blair, "Real-time DNA microarrays: Reality check," Biochem. Soc. Trans., vol. 37, no. 2, pp. 471-475, Apr. 2009.

[3] S. Park and U. Krull, "A spatially resolved nucleic acid biochip based on a gradient of density of immobilized probe oligonucleotide," Anal. Chim. Acta, vol. 564, no. 2, pp. 133-140, Apr. 2006.

[4] M. Schena, D. Shalon, R. Davis, and P. Brown, "Quantitative monitoring of gene expression patterns with a complementary DNA microarray," Science, vol. 270, no. 5235, pp. 467-470, Oct. 1995.

[5] K. Murari, R. Cummings, N. Thakor, and G. Gauwenberghs, "A CMOS in-pixel CTIA high-sensitivity fluorescence imager," IEEE Trans. Biomed. Circuits Syst., vol. 5, no. 5, pp. 449-458, Oct. 2011.
[6] N. Nelson, D. Sander, M. Dandin, S. Prakash, A. Sarje, and P. Abshire, "Handheld fluorometers for lab-on-a-chip applications," IEEE Trans. Biomed. Circuits Syst., vol. 3, no. 2, pp. 97-107, Apr. 2009.

[7] S. Kong, D. Wijngaards, and R. Wolffenbuttel, "Infrared micro-spectrometer based on a diffraction grating," Sens. Actuators A, no. 92, pp. 88-95, Aug. 2001.

[8] J. Correia, G. Graaf, M. Bartek, and R. Wolffenbuttel, "A single-chip CMOS optical microspectrometer with light-to-frequency converter and bus interface," IEEE J. Solid-State Circuits, vol. 37, no. 10, pp. 1344-1347, Oct. 2002.

[9] X. Fang, V. Hsiao, V. Chodavarapu, A. Titus, and A. Cartwright, "Colorimetric porous photonic bandgap sensors with integrated CMOS color detectors," IEEE Sensors J., vol. 6, no. 3, pp. 661-667, Jun. 2006.

[10] C. Richard, T. Courcier, P. Pittet, S. Martel, L. Ouellet, G. Lu, V. Aimez, and P. Charette, "CMOS buried quad p-n junction photodetector for multi-wavelength analysis," Opt. Express, vol. 20, no. 3, pp. 2053-2061, Jan. 2012.

[11] P. Hubel, J. Liu, and R. Guttosch, "Spatial frequency response of color image sensors: Bayer color filters and Foveon X3," Proc. SPIE, vol. 53 , no. 1 , pp. 402-407, 2004.

[12] H. Ishii, Y. Maruyama, H. Takao, M. Ishida, and K. Sawada, "Improvement in filter-less fluorescence sensor capability by optimization of potential distribution," in Proc. 4th Asia-Pacific Conf. Transducers and Micro-Nano Technology, Jun. 2008, pp. 68-71.

[13] J. Choi, G. Beaucage, J. Nevin, J. Lee, A. Puntambekar, and J. Lee, "Disposable smart lab on a chip for point-of-care clinical diagnostics," Proc. IEEE, vol. 92, no. 1, pp. 154-173, Jan. 2004.

[14] J. Honghao, D. Sander, A. Haas, and P. Abshire, "Contact imaging: Simulation and experiment," IEEE Trans. Circuits Syst. I, Reg. Papers, vol. 54, no. 8, pp. 1698-1710, Aug. 2007.

[15] D. Daivasagaya, L. Yao, K. Yung, M. Hajj-Hassan, M. Cheung, V. Chodavarapu, and F. Bright, "Contact CMOS imaging of gaseous oxygen sensor array," Sens Actuators B, vol. 157, no. 2, pp. 408-416, Oct. 2011.

[16] C. Richard, A. Renaudin, V. Aimez, and P. Charette, "An integrated hybrid interference and absorption filter for fluorescence detection in lab-on-a-chip devices," Lab on a Chip, vol. 9, no. 10, pp. 1371-1376, May 2009

[17] D. Ho, G. Gulak, and R. Genov, "CMOS field-modulated color sensor," in Proc. IEEE Custom Integrated Circuit Conf., Sep. 2011.

[18] D. Ho, M. Noor, U. Krull, G. Gulak, and R. Genov, "Single-filter multicolor CMOS fluorescent contact sensing microsystem," in Proc. IEEE Int. Symp. Circuits and Systems, May 2012.

[19] D. Ho, M. Noor, U. Krull, G. Gulak, and R. Genov, "CMOS color photogate sensor," IEEE Trans. Biomed. Circuits Syst., accepted for publication.

[20] H. Ai, K. Hazelwood, M. Davidson, and R. Campbell, "Fluorescent protein FRET pairs for ratiometric imaging of dual biosensors," Nature Methods, vol. 5, no. 5, pp. 401-403, Apr. 2008.

[21] M. Bruchez, M. Moronne, P. Gin, S. Weiss, and A. Alivisatos, "Semiconductor nanocrystals as fluorescent biological labels," Science, vol. 281, no. 5385, pp. 2013-2016, Sep. 1998.

[22] Integrated DNA technologies, Jun. 1, 2012 [Online]. Available: http:// www.idtdna.com

[23] X. Peng, H. Chen, D. Draney, A. Schutz-Geschwender, and D. Olive, "A nonfluorescent, broad range quencher dye for forster resonance energy transfer assays," Anal. Biochem., vol. 388, no. 2, pp. 220-228, May 2009.

[24] H. Tian, B. Fowler, and A. E. Gamal, "Analysis of temporal noise in CMOS photodiode active pixel sensor," IEEE J. Solid-State Circuits, vol. 36, no. 1, pp. 92-101, Jan. 2001.

[25] J. Kabuss and M. Richter, "Theory of light scattering from semiconductor quantum dots: Excitation frequency dependent emission dynamics," Photon. Nanostructures, vol. 9, no. 1, pp. 296-301, Oct. 2011.

[26] M. Neumann and D. Gabel, "Simple method for reduction of autofluorescence in fluorescence microscopy," Nature Biotechnol., vol. 50, no. 3, pp. 437-439, Mar. 2002.

[27] M. Munson, M. Hasenbank, E. Fu, and P. Yager, "Suppression of nonspecific absorption using sheath flow," Nature Biotechnol., vol. 4, no. 1, pp. 438-445, Sep. 2004.

[28] G. Long and J. Winefordner, "Limit of detection a closer look at the IUPAC definition," Anal. Chem., vol. 55, no. 7, pp. 712A-724A, Jul. 1983.

[29] Philips, Sep. 7, 2011 [Online]. Available: http://www.philipslumileds.com

[30] Omega Optical, May 8, 2011 [Online]. Available: http://www. omegafilters.com 
[31] Cytodiagnostics, Jun. 1, 2012 [Online]. Available: http://www.cytodiagnostics.com

[32] A. Tavares, M. Noor, W. Algar, C. Vannoy, C. Lu, and U. Krull, "Toward an on-chip multiplexed nucleic acid hybridization assay using immobilized quantum dot-oligonucleotide conjugates and fluorescence resonance energy transfer," Proc. SPIE: Colloidal Quantum Dots/Nanocrystals for Biomedical Applicat., vol. 7909, no. 79090X, pp. 133-141, Jan. 2011.

[33] C. Leatherdale, W. Woo, F. Mikulec, and M. Bawendi, "On the absorption cross section of CdSe nanocrystal quantum dots," J. Phys. Chem. $B$, vol. 106, no. 31, pp. 7619-7622, Jul. 2002.

[34] W. Algar, D. Wegner, A. Huston, J. Blanco-Canosa, M. Stewart, A. Armstrong, P. Dawson, N. Hildebrandt, and I. Medintz, "Quantum dots as simultaneous acceptors and donors in time-gated forster resonance energy transfer relays: Characterization and biosensing," $J$. Amer. Chem. Soc., vol. 134, no. 3, pp. 1876-1891, Jan. 2012.

[35] W. Algar and U. Krull, "Towards multi-colour strategies for the detection of oligonucleotide hybridization using quantum dots as energy donors in fluorescence resonance energy transfer (FRET)," Anal. Chim. Acta, vol. 581, no. 2, pp. 193-201, Jan. 2007.

[36] H. Wong, "Technology and device scaling considerations for CMOS imagers," IEEE Trans. Electron Devices, vol. 43, no. 12, pp. 2131-2142, Dec. 1996.

[37] K. Peck, L. Stryer, A. Glazer, and R. Mathies, "Single-molecule fluorescence detection: Autocorrelation criterion and experimental realization with phycoerythrin," Proc. Nat. Acad. Sci., vol. 86, no. 1, pp. 4087-4091, Feb. 1989

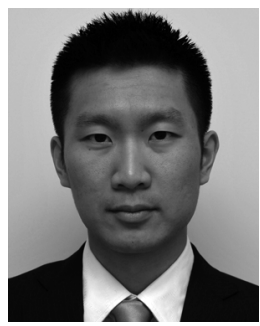

Derek Ho (S'09) received the B.A.Sc. (first class) and M.S. degrees from the University of British Columbia, Vancouver, BC, Canada, in 2005 and 2007, respectively.

Currently, he is working toward the Ph.D. degree in the Department of Electrical and Computer Engineering, University of Toronto, Toronto, ON, Canada. His research interests are in mixed-signal VLSI circuits and systems for sensory acquisition and processing with emphasis on spectral and wide-dynamic range imaging, photonic lab-on-a-chip, point-of-care screening, and biochemical detection applications.

Mr. Ho was the recipient of the Postgraduate Scholarship and Undergraduate Student Research Award from the Natural Sciences and Engineering Research Council of Canada. He was also awarded the Ontario Graduate Scholarship in Science and Technology, and the Mary H. Beatty Fellowship.

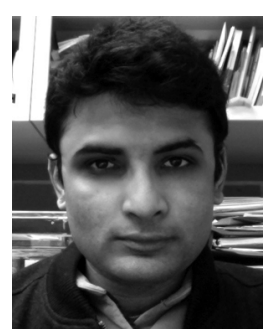

M. Omair Noor received the Hon. B.Sc. degree in the biotechnology specialist program and the M.Sc. degree in bioanalytical chemistry from the University of Toronto Mississauga, Mississauga, ON, Canada, in 2008 and 2010, respectively.

Currently, he is working toward the Ph.D. degree in the Department of Chemistry at the University of Toronto, Toronto, ON, Canada, with the Chemical Sensors Group under the supervision of Prof. Ulrich J. Krull. His research interests focus on the integration of quantum dots and FRET based assays for nucleic acid detection using microfluidic channels and paper as solid supports.

Mr. Noor holds an Ontario Graduate Scholarship (OGS) from the Ontario Ministry of Training, Colleges and Universities (MTCU). He was also awarded the Undergraduate Student Research Award (USRA) from the Natural Sciences and Engineering Research Council of Canada (NSERC).

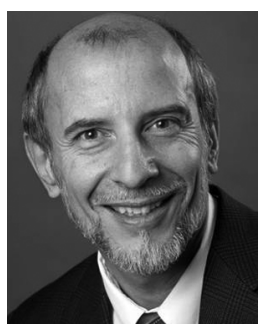

Ulrich J. Krull is appointed as a Professor of Analytical Chemistry at the University of Toronto, Toronto, ON, Canada, and holds the endowed AstraZeneca Chair in Biotechnology.

His research interests are in the areas of biosensor and diagnostic technologies, and applications to biotechnology, forensic, clinical and environmental chemistry. His research work is exploring the use of nanoscale materials and microfluidics technologies to build devices for detection of DNA and RNA targets. Prof. Krull is an editor for Analytica Chimica $A c t a$, and serves on a number of scientific advisory boards for industry.

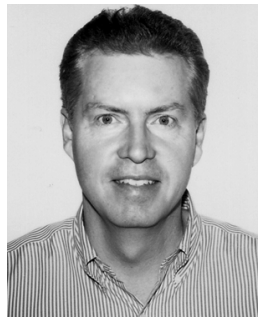

Glenn Gulak (M'90-SM'96) received the Ph.D. degree from the University of Manitoba, Winnipeg, MB, Canada, while holding a Natural Sciences and Engineering Research Council of Canada Postgraduate Scholarship.

$\mathrm{He}$ is a Professor in the Department of Electrical and Computer Engineering at the University of Toronto, Toronto, ON, Canada. He is a registered Professional Engineer in the Province of Ontario. His present research interests are in the areas of algorithms, circuits, and CMOS system-on-chip implementations for digital communication systems and, additionally, in the area of CMOS biosensors. His current research projects are focused on high-performance MIMO OFDM implementations and in CMOS biosensors. He has authored or coauthored more than 100 publications in refereed journal and refereed conference proceedings. He has received numerous teaching awards for undergraduate courses taught in both the Department of Computer Science and the Department of Electrical and Computer Engineering at the University of Toronto. He held the L. Lau Chair in Electrical and Computer Engineering for the 5-year term from 1999 to 2004. From March 2001 to March 2003 he was the Chief Technical Officer and Senior VP LSI Engineering of a fabless semiconductor startup headquartered in Irvine, CA, USA. From January 1985 to January 1988 he was a Research Associate in the Information Systems Laboratory and the Computer Systems Laboratory at Stanford University, Stanford, CA, USA. He holds the Canada Research Chair in Signal Processing Systems and the Edward S. Rogers Sr. Chair in Electrical Engineering.

Dr. Gulak has served on the ISSCC Signal Processing Technical Subcommittee from 1990 to 1999, ISSCC Technical Vice-Chair in 2000 and served as the Technical Program Chair for ISSCC 2001. He received the IEEE Millenium Medal in 2001. He served on the Technology Directions Subcommittee for ISSCC from 2005 to 2008. He currently serves as the Chair of the Publications Committee for the IEEE Solid-State Circuits Society.

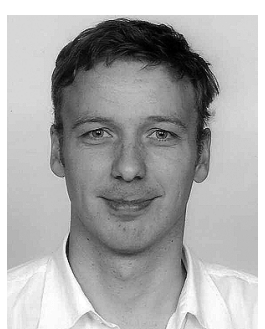

Roman Genov (S'96-M'02-SM'11) received the B.S. degree in electrical engineering from the Rochester Institute of Technology, Rochester, NY, USA, in 1996, and the M.S.E. and Ph.D. degrees in electrical and computer engineering from The Johns Hopkins University, Baltimore, MD, USA, in 1998 and 2002, respectively.

He has held engineering positions at Atmel Corporation, Columbia, MD, USA, in 1995 and Xerox Corporation, Rochester, NY, USA, in 1996. He was a Visiting Researcher in the Laboratory of Intelligent Systems at the Swiss Federal Institute of Technology (EPFL), Lausanne, Switzerland, in 1998 and in the Center for Biological and Computational Learning at the Massachusetts Institute of Technology, Cambridge, MA, USA, in 1999. He is currently an Associate Professor in the Department of Electrical and Computer Engineering, University of Toronto, Toronto, ON, Canada. His research interests include analog and digital VLSI circuits, systems and algorithms for energy-efficient signal processing with applications to electrical, chemical and photonic sensory information acquisition, biosensor arrays, brain-silicon interfaces, parallel signal processing, adaptive computing for pattern recognition, and implantable and wearable biomedical electronics.

Dr. Genov is a corecipient of Best Paper Award of the IEEE Biomedical Circuits and Systems Conference, Best Student Paper Award of the IEEE International Symposium on Circuits and Systems, Best Paper Award of the IEEE Circuits and Systems Society Sensory Systems Technical Committee, Brian L. Barge Award for Excellence in Microsystems Integration, MEMSCAP Microsystems Design Award, the DALSA Corporation Award for Excellence in Microsystems Innovation, and the Canadian Institutes of Health Research Next Generation Award. He was a Technical Program Co-Chair at the IEEE Biomedical Circuits and Systems Conference. He was an Associate Editor of IEEE TRANSACTIONS ON CIRCUITS AND SYSTEMS II: EXPRESS BRIEFS and IEEE Signal Processing Letters. He is currently an Associate Editor of IEEE TRANSACTIONS ON BIOMEDICAL CIRCUITS AND SYSTEMS. 